\title{
Empirical calculations of interactions between two mesogenic molecules
}

\author{
D. Sy and M. Ptak \\ Centre de Biophysique Moléculaire, C.N.R.S., 45045 Orléans Cedex, France \\ and U.E.R. Sciences, Université Orléans, 45045 Orléans Cedex, France
}

(Reçu le 19 décembre 1978, accepté le 23 janvier 1979)

\begin{abstract}
Résumé. - Des méthodes empiriques de calcul sont utilisées pour calculer les interactions atomiques entre deux molécules rigides d'un smectogène. Les configurations stables sont étudiées et sont reliées à la possibilité d'existence d'une phase de type smectique où les mouvements longitudinaux et axiaux n'auraient que de faibles amplitudes. Les interactions de Van der Waals jouent un rôle prédominant pour stabiliser le dimère.
\end{abstract}

\begin{abstract}
The interactions between two rigid molecules are calculated by empirical methods based on atomic potential contributions. The stable configurations are studied and related to the possible existence of a smecticlike phase with translational and rotational restricted motions. The Van der Waals interactions are shown to be determinant in the dimer stabilization.
\end{abstract}

1. Introduction. - The Maier and Saupe theory of nematic phases [1] and the MacMillan theory of smectic phases [2] are classical examples of microscopic theories developed to account for relations between physical properties and molecular structures of mesophases. Most such theories are based on oversimplified molecular models, such as rodlike bodies, in a mean field which results from steric or dipole-dipole interactions. An atomic scale analysis of intraand intermolecular forces acting on molecules in mesophases is extremely difficult because of complicated averaging effects due to intra- and intermolecular motions. On the contrary, such an analysis seems quite possible in crystal structures of mesogenic compounds. The determination of these crystal structures as precursors of liquid crystal structures is of present interest [3]. This paper reports such an approach based on interaction calculations classically used in conformational analysis [4]. We examine a dimer of a smectogen : ethyl p-methoxybenzilidene aminocinnamate (MBACE), the crystal structure of which was recently determined [5]. In the present step, we use empirical potentials and consider both molecules as rigid interacting bodies. With these approximations, stable arrangements and predominant contributions to the interaction energy are studied.

2. Method. - First, the stable conformation of an isolated molecule is determined by minimization of the intramolecular energy on the internal torsion angles. Interactions between two molecules in their minimized conformation are then calculated. Empirical atomic potentials, including electrostatic, Van der Waals and, for intramolecular energy, torsional terms are used. Partial atomic charges are calculated by Del Ré [6] and Hückel [7] methods for $\sigma$ and $\pi$ charges respectively (Fig. 1). For a check,

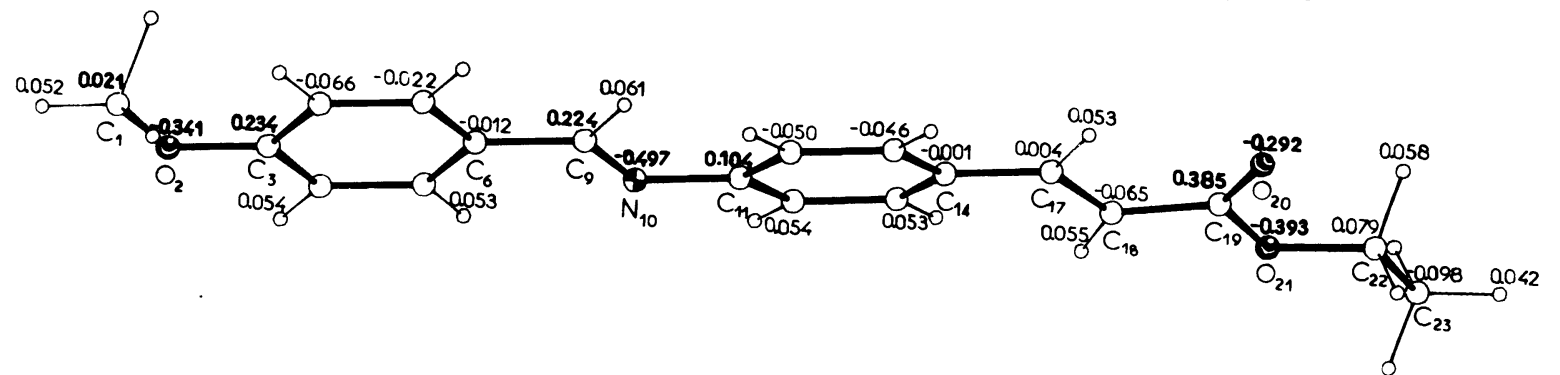

Fig. 1. - MBACE molecule. Atoms numbering and partial atomic charges in fraction of the electron charge (charges corresponding to the three local dipoles are displayed in bold type). 
the dipole moment of anisole, as a fragment of the MBACE molecule, is calculated : $1.50 \mathrm{D}$, comparable to the experimental value in solution $1.25 \mathrm{D}$. The agreement is not so good for the conjugated fragments. The value of the dielectric permittivity is taken to be 1 . However, it will be shown that the electrostatic contribution is small and low weights are given to the actual values of the charge distribution. A Buckingham exponential type potential represents Van der Waals interactions; the coefficients are those of Liquori [8]. The torsional potential is periodic and the values of the barrier heights around $\mathrm{N}_{10}-\mathrm{C}_{11}$ and $\mathrm{C}_{14}-\mathrm{C}_{17}$ bonds are 20.3 and $2.8 \mathrm{kcal} / \mathrm{mol}$. [9].

The molecular geometry is defined by an arbitrary $z$ axis $\left(\mathrm{O}_{2}, \mathrm{C}_{18}\right)$ and a plane $\left(\mathrm{O}_{2}, \mathrm{C}_{9}, \mathrm{C}_{18}\right)$. A moving molecule is related to the reference molecule through an inversion centre $(\uparrow \downarrow)$ or by a translation ( $\uparrow)$. Four parameters are required to determine the relative position of two molecules with parallel axes as shown on figure 2. Each molecule is assumed to remain in its minimized conformation; in other words, we suppose that, in an isolated dimer, intermolecular interactions do not significantly modify the individual molecular conformations.

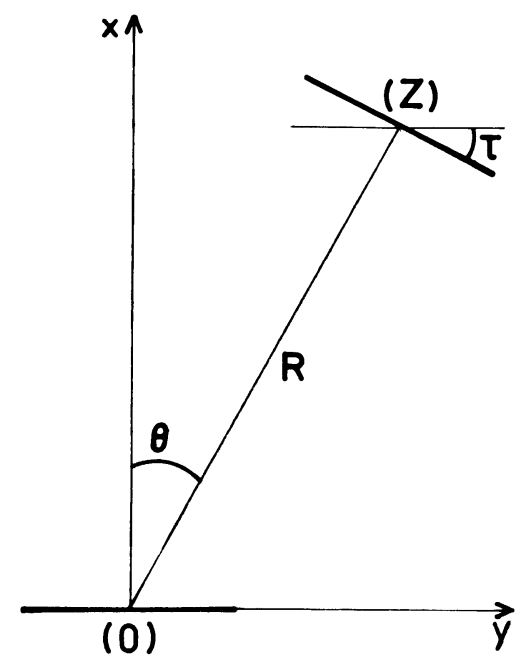

Fig. 2. - Geometrical parameters for a pair of molecules. The reference plane of each molecule, viewed along its reference axis, is represented by a segment. $Z$ is the distance on the $z$ axis between the oxygen atoms $\mathrm{O}_{2}$ of each methoxy group.

3. Results. - 3.1 INTRAMOLECULAR ENERGY MINIMIZATION FOR A FREE MOLECULE. - The molecular conformation mainly depends on two torsion angles : the torsion around the $\mathrm{N}_{10}-\mathrm{C}_{11}$ bond determines the molecular planarity and the torsion around the $\mathrm{C}_{14}-\mathrm{C}_{17}$ bond determines the position of the chain with respect to the aromatic core, that is, the molecular linearity. Charge conjugation and the high value of the N-C rotation barrier should favor a planar conformation; however, because of steric hindrance between $\mathrm{H}$ atoms bonded to $\mathrm{C}_{9}$ and $\mathrm{C}_{12}$, the minimized conformation shows some deviation from planarity. The optimized values of the torsion angles for the free molecule of MBACE, and those found in the crystal structures of MBACE [5] and of its homologous MBAMCP [10] (terminal chain $\alpha$-methyl-n-propyl), are reported in table $I$; it appears that the crystalline field significantly influences the molecular conformation [11].

Table I. - Molecular conformation.

$\begin{array}{cccc}\begin{array}{c}\text { Torsion } \\ \text { angle }\end{array} & \begin{array}{c}\text { Crystal structure } \\ \text { MBAMCP }\end{array} & \begin{array}{c}\text { Crystal structure } \\ \text { MBACE }\end{array} & \begin{array}{c}\text { Free molecule } \\ \text { MBACE }\end{array} \\ - & - & - & - \\ \mathrm{N}_{10}-\mathrm{C}_{11} & -36.1^{\circ} & -8.7^{\circ} & -14.8^{\circ} \\ \mathrm{C}_{14}-\mathrm{C}_{17} & +20.8^{\circ} & +5.1^{\circ} & +16.0^{\circ}\end{array}$

3.2 STATIC INTERACTIONS BETWEEN TWO MOLECULES WITH PARALLEL REFERENCE PLANES $\left(\tau=0^{\circ}\right)$ AND PARAllel OR ANTIPARALlel AXES. - Preliminary calculations show that the interaction energy decreases with a tilt between the axes of both molecules. The study was then restricted to a pair of molecules $\uparrow \uparrow$ or $\uparrow \downarrow$.

For a given set $(R, \theta)$, relative molecular translation along $z$ axis is achieved and intermolecular energy calculated as a function of $Z$. For superposed molecules $\left(\theta=0^{\circ}\right)$, at short distances $R$, five potential wells can be seen in figure 3. As $R$ increases, one of

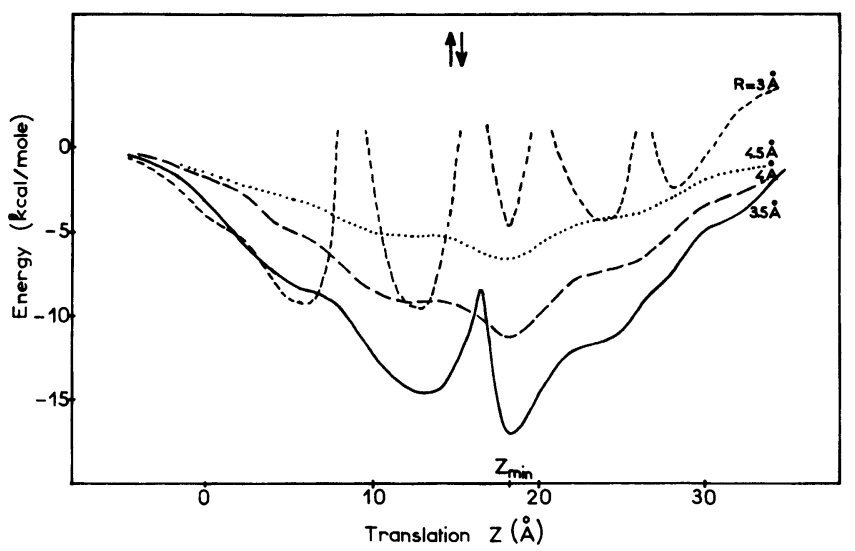

a)

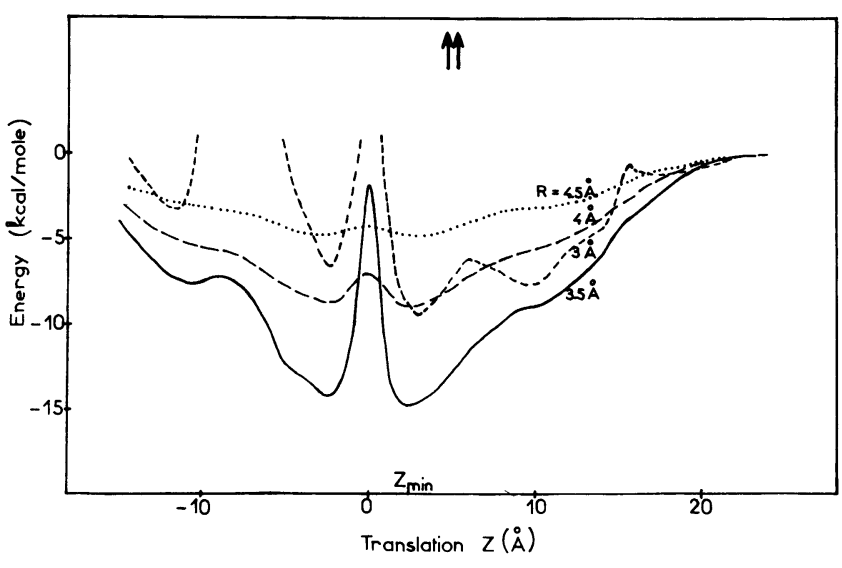

b)

Fig. 3. - Translational intermolecular energy dependence for superposed molecules $\left(\theta=0^{\circ}, \tau=0^{\circ}\right)$ parallel $(\uparrow \uparrow)$ and antiparallel $(\uparrow \downarrow)$. 

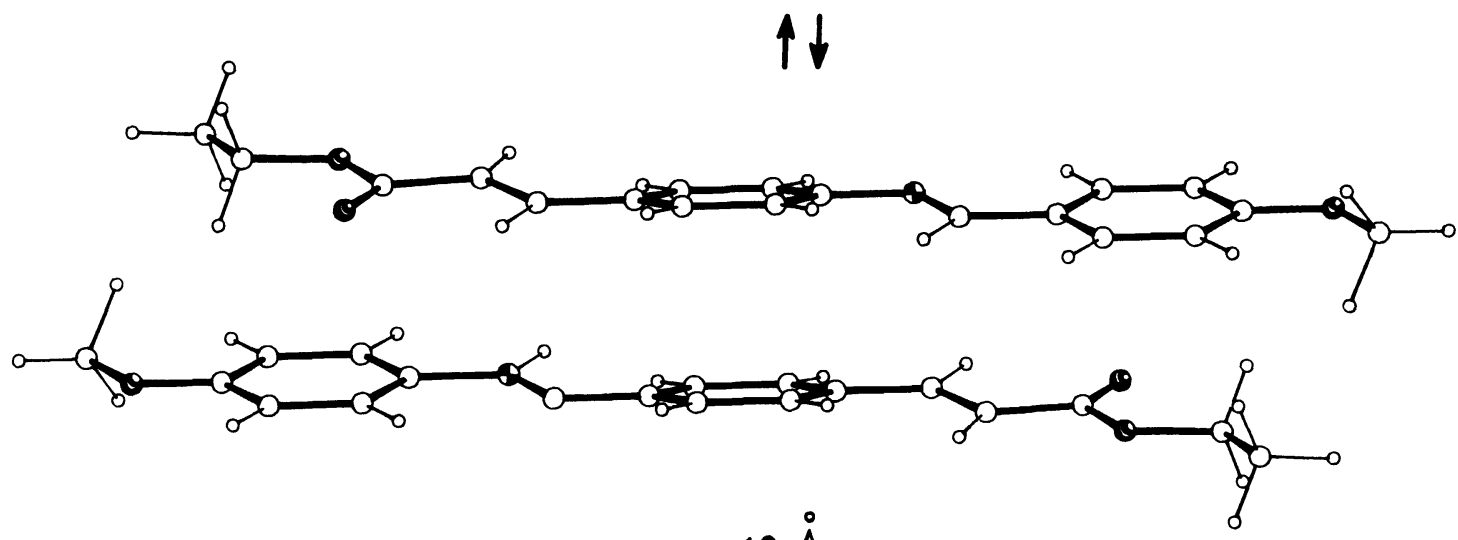

$10 \AA$

\section{$\uparrow \uparrow$}
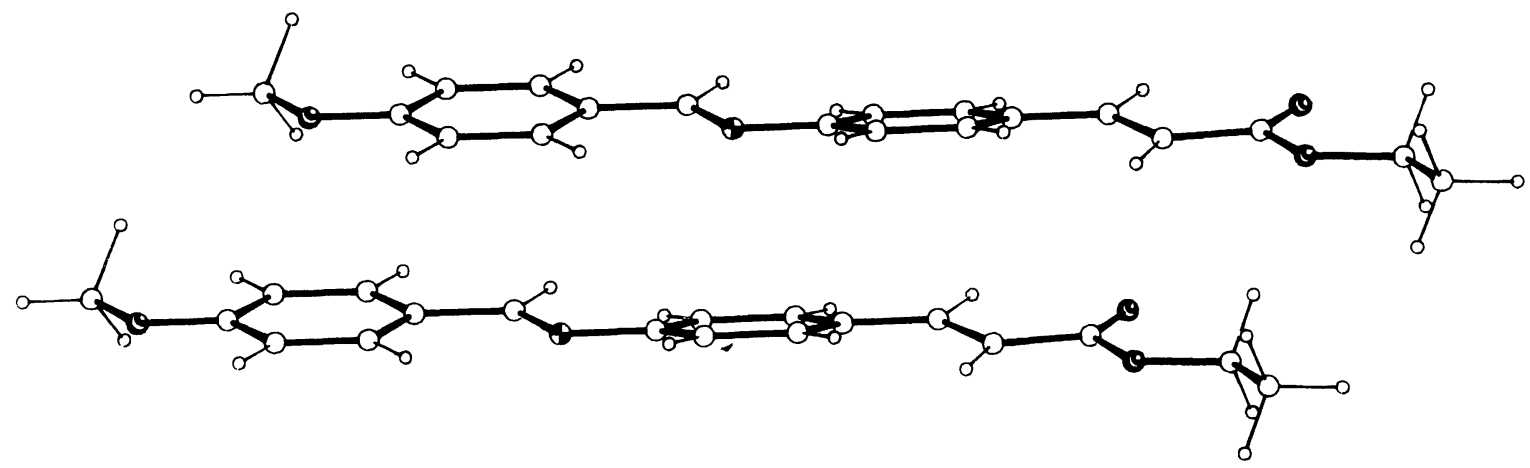

Fig. 4. - Stable arrangements for a pair of superposed molecules : $\uparrow R_{\min }=3.3 \AA, Z_{\min }=2.7 \AA$ and $\uparrow \downarrow R_{\min }=3.4 \AA, Z_{\min }=18.2 \AA$.

the minima is selected which corresponds to an overlap of both molecules $Z_{\min } \simeq 18 \AA(\uparrow \downarrow)$ and $Z_{\text {min }} \simeq 3 \AA(\uparrow \uparrow)$; the interaction energy is of the same order of magnitude for both arrangements represented in figure 4 . For further increase of $R$, the interaction decreases and the minimum gets broad. For $\theta$ variations in the whole range $0^{\circ}$ to $360^{\circ}$, the values of $Z_{\min }$ do not depart from the previous ones by more than $2 \AA$. The values of $R$, which minimize the intermolecular energy, $R_{\min }$, are plotted in
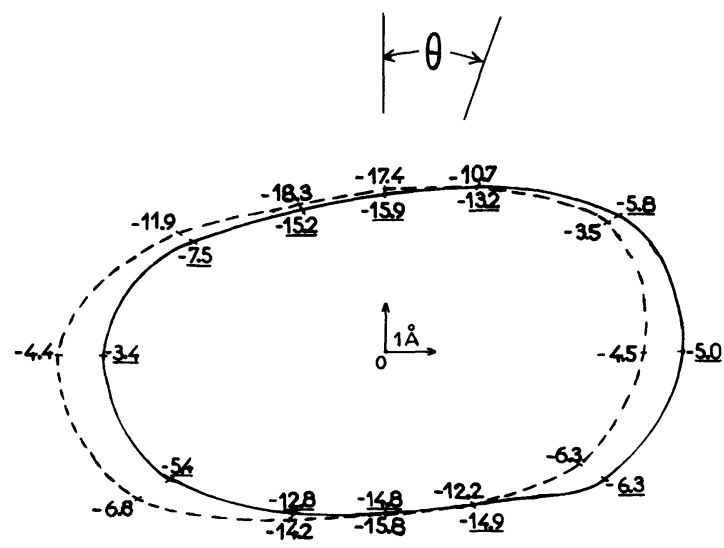

Fig. 5. - Polar diagram of relative distances and corresponding intermolecular energies (in $\mathrm{kcal} / \mathrm{mol}$.) for stable arrangements. $\frac{1}{-\underline{15.9}}$ parallel molecules $(\uparrow \uparrow)--17.4$ - antiparallel molecules $(\uparrow \downarrow)$ polar coordinates in figure 5. This diagram reflects the geometrical form of the molecular cross-section for two quasi-coplanar phenyl rings. The energy is larger for short $R_{\min }\left(\theta=0^{\circ}\right)$ and weaker for large $R_{\min }\left(\theta=90^{\circ}\right)$. In any case, the contribution of the electrostatic interaction to the total energy is small (table II).

3.3 INTERACTION BETWEEN TWO MOLECULES ROTATED AROUND THEIR AXES. - Independent rotation of both molecules is achieved by keeping one molecule fixed and varying the angular position $(\theta)$ and reference plane $(\tau)$ of the other one. $Z$ is fixed for any $\theta$ at the averaged value $Z=17 \AA$ for $\uparrow \downarrow$ molecules and the energy calculated as a function of $\tau$ for different values of $\theta$ and $R$. The curves exhibit a barrier, the height of which can be ajusted by a convenient choice of the intermolecular distance $R$; its position and width are $\theta$ dependent. On figure 6 are plotted the curves $E(\tau)$ for different values of $\theta$ in the range $0^{\circ}$ to $90^{\circ}$ and corresponding $R$ values in the range 4.7 to $5.9 \AA$. The ranges of forbidden values of $\tau$ for a given $\theta$ are sketched on figure 7. It is clear that axial molecular rotations are restricted or strongly correlated between neighbour molecules.

4. Discussion. - Inspection of molecular models makes some of the previous results understandable : 
Table II. - Intermolecular energy ( $\mathrm{kcal} / \mathrm{mol}$.$) .$

\begin{tabular}{lcccc} 
& \multicolumn{1}{c}{$\theta=0^{\circ}$} & \multicolumn{1}{c}{$\theta=90^{\circ}$} \\
& $R_{\min }=3.3 \AA$ & $R_{\min }=3.4 \AA$ & $R_{\min }=6.15 \AA$ & $R_{\min }=5.4 \AA$ \\
$Z_{\min }=2.7 \AA$ & $Z_{\min }=18.2 \AA$ & $Z_{\min }=3.2 \AA$ & $Z_{\min }=16.8 \AA$ \\
& - & - & - & - \\
Electrostatic energy & +1.40 & -0.64 & -0.83 & -0.51 \\
Van der Waals energy & -17.27 & -16.75 & -4.21 & -3.97 \\
Total energy & -15.87 & -17.39 & -5.05 & -4.49
\end{tabular}

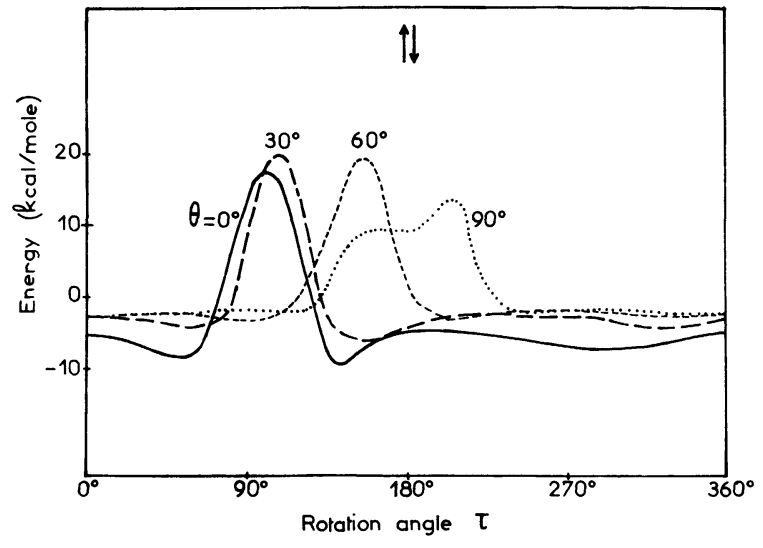

Fig. 6. - Rotational intermolecular energy dependence for antiparallel molecules $Z=17 \AA\left(R=4.7,5.4,5.9,5.85 \AA\right.$ for $\theta=0^{\circ}$, $30^{\circ}, 60^{\circ}, 90^{\circ}$.

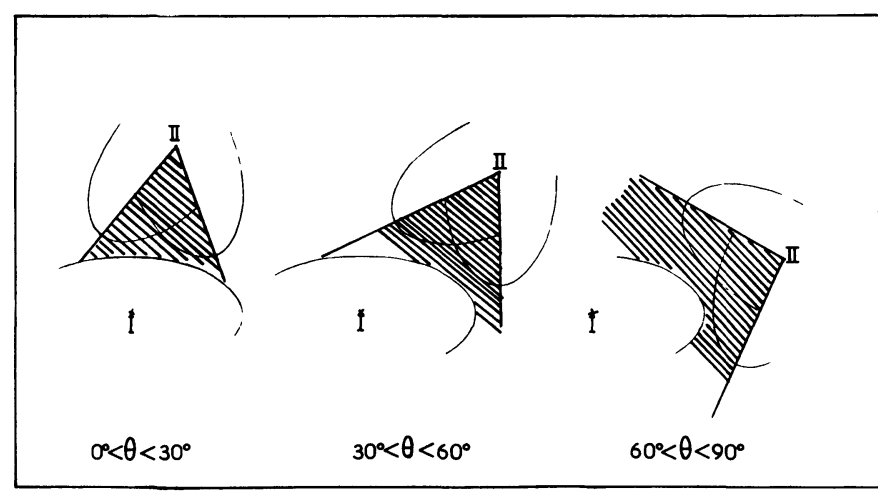

Fig. 7. - Schematic representation of rotational hindrance. Ellipses represent the cross-section of the first benzene ring.

maximum interaction for stacked molecules, angular dependence of intermolecular distances and corresponding energies, strong restrictions to axial rotations. Further features emerged from the quantitative study. The translational energy dependence at short distances $R$ shows discrete possible configurations corresponding to stacking of phenyl or ester groups with intercalated dipoles, The Van der Waals term in the potential is determinant in such interactions. The crystalline field can select among these various arrangements and this could explain the polymorphism of MBACE [12]. $\uparrow \uparrow$ and $\uparrow \downarrow$ pairs nearly have the same stability, maximum for $R \simeq 3.5 \AA$, superposed and overlapping molecules. This association could represent a compact crystalline mode. In fact, such dimers appear as structural units in some mesogenic crystal structures [3] and could remain as such in mesophases [13]. It should be of interest to know the structure of another crystalline form, especially that obtained by cooling the smectic B mesophase. For larger intermolecular distances up to $5 \AA$, as in smectic phases, and various angular positions, the selected configurations are nearly those of maximum overlap; the real value of $Z_{\min }$ should be compatible with minimum steric interaction and relative minimum for electrostatic. Despite its low contribution, this last interaction may play a selective role : it can be noted for example that, for the $\uparrow \uparrow$ arrangement, the longitudinal components of both molecular dipole moments, interacting in an unfavourable way at $Z=0 \AA$ for any $\theta$ value, contribute to a slipping of the molecules.

Further increase in $R$ releases translational and rotational motions as in nematic phases.

5. Conclusion. - This study on a simplified model of two rigid interacting molecules clearly appears more significant than a mere molecular models manipulation. It shows the predominant role of Van der Waals interactions as compared with electrostatic interaction. It gives a good basis to predict the stable configurations for a large set of molecules and the existence of a phase where all the molecular are stacked, with maximum overlap, in layers. The other structural characteristics of such a phase are $\uparrow \uparrow$ or $\uparrow \downarrow$ arrangements with comparable interaction energies, mean intermolecular distances according to the angular position of the order of $5 \AA$, restricted translational motions and correlated or hindered rotations. That is in qualitative agreement with the classical description of a non tilted smectic mesophase. It suggests the actual possibility of atomic level analysis for intermolecular interactions in smectics. Further improvements of the crude model are clearly required. More refined semi-empirical or quantum energy calculations are to be used; the molecular conformation must be adjusted during the intermolecular energy minimization and not independently ; more than two molecules must be considered ; statistical methods are to be used to account for intramolecular motions (especially for molecules with long flexible aliphatic chains [14]) and dynamical molecular motions with angular correlation. One can expect then to get a valuable theory for low temperature mesophases. 


\section{References}

[1] Maier, W. and SAupe, A., Z. Naturforsch. A 15 (1960) 287.

[2] McMillan, W. L., Phys. Rev. A 4 (1971) 1238. McMillan, W. L., Phys. Rev. A 8 (1973) 1921.

[3] Bryan, R. F., Communication to the 7th Intern. Conf. on Liquid Cryst., Bordeaux (1978), J. Physique Colloq. 40 (1979) and references therein.

[4] Kitaigorodsky, A. I., Molecular Crystals and Molecules (Academic Press, New York) 1973.

[5] Cotrait, M. and Pesquer, M., Acta Cryst. B 23 (1977) 2826.

[6] Del Re, G., J. Chem. Soc. (1958) 4031.

[7] Pullman, A. and Pullman, B., Quantum Biochemistry (Interscience, New York) 1963.

[8] Liquori, A. M., Damiani, A. and de Coen, J. L., J. Mol. Biol. 33 (1968) 445.
[9] Perricaudet, M. and Pullman, A., Int. J. Pept. Protein Res. 5 (1973) 99.

Yan, J. F., van Der Kool, G. and Scheraga, H. A., J. Chem. Phys. 49 (1968) 2713.

[10] Cotrait, M., Sy, D. and Ptak, M., Acta Cryst. B 31 (1975) 1869.

[11] Bernstein, J. and Hagler, A. T., J. Am. Chem. Soc. 100 (1978) 673.

[12] Chistyakov, I. G., Schabischev, L. S., Jarenov, R. I. and Gusakova, L. A., Mol. Cryst. Liq. Cryst. 7 (1969) 279.

[13] Krigbaum, W. R., Poirier, J. C. and Costello, M. J., Mol. Cryst. Liq. Cryst. 20 (1973) 133.

[14] MarcelJa, S., Solid State Commun. 13 (1973) 759. 